\title{
Genetic Rodent Models of Amyotrophic Lateral Sclerosis
}

\author{
L. Van Den Bosch \\ Neurobiology, Vesalius Research Center, K.U. Leuven and VIB, Campus Gasthuisberg O\&N2 PB1022, Herestraat 49, \\ 3000 Leuven, Belgium
}

Correspondence should be addressed to L. Van Den Bosch, ludo.vandenbosch@vib-kuleuven.be

Received 15 September 2010; Accepted 29 November 2010

Academic Editor: Oreste Gualillo

Copyright () 2011 L. Van Den Bosch. This is an open access article distributed under the Creative Commons Attribution License, which permits unrestricted use, distribution, and reproduction in any medium, provided the original work is properly cited.

\begin{abstract}
Amyotrophic lateral sclerosis (ALS) is a neurodegenerative disorder characterized by the selective death of motor neurons in the motor cortex, brainstem, and spinal cord. A large number of rodent models are available that show motor neuron death and a progressive motor phenotype that is more or less reminiscent of what occurs in patients. These rodent models contain genes with spontaneous or induced mutations or (over) express different (mutant) genes. Some of these models have been of great value to delineate potential pathogenic mechanisms that cause and/or modulate selective motor neuron degeneration. In addition, these genetic rodent models play a crucial role in testing and selecting potential therapeutics that can be used to treat ALS and/or other motor neuron disorders. In this paper, we give a systematic overview of the most important genetic rodent models that show motor neuron degeneration and/or develop a motor phenotype. In addition, we discuss the value and limitations of the different models and conclude that it remains a challenge to find more and better rodent models based on mutations in new genes causing ALS.
\end{abstract}

\section{Introduction}

Amyotrophic lateral sclerosis (ALS) is a neurodegenerative disease characterized by the selective death of motor neurons in the motor cortex, brainstem, and spinal cord. Typically, ALS is an adult-onset disorder with a fast progression. About $5 \%$ to $10 \%$ of ALS patients have a family history (FALS), and most of these patients inherit the disease in an autosomal dominant way. The large majority of patients (90\% to 95\%) have no clear family history and are considered as sporadic ALS (SALS). FALS and SALS are clinically indistinguishable, and all patients experience muscle weakness, atrophy, and spasticity. This is the consequence of the loss of both upper and lower motor neurons. Ultimately, patients become paralyzed and denervation of respiratory muscles causes the death of the patient, on average three to five years after the first clinical signs. As studying a sporadic disease is very difficult, most research focused on the genetic causes of FALS (for reviews see [1-3]).

The most important and most studied cause of FALS are mutations in the gene encoding superoxide dismutase 1 (SOD1). On average, SOD1 mutations are responsible for about $20 \%$ of FALS cases [4]. Recently, mutations in the genes encoding the TAR DNA-binding protein 43 (TDP-43;
[5-9]) and FUS/TLS [10, 11] were identified as important gene defects causing typical FALS in approximately $5 \%-10 \%$ of the familial cases.

All other genetic causes of FALS are rare and/or cause an atypical form of ALS. These include mutations in the genes encoding alsin (ALS2; [12]), senataxin (ALS4; [13]), spatacsin (ALS5; [14]), vesicle-associated membrane protein B (VAPB; ALS8; [15]), angiogenin (ALS9; [16]), optineurin (ALS12; [17]), and dynactin [18]. In addition, (rare) sequence variants in a number of other genes were reported to be associated with ALS. These variations were found in candidate genes or through genome wide association studies [2]. In all cases, it is unclear whether these genes and/or variations play a role in ALS, and these associations remain to be confirmed.

For years, a number of mouse models exist that contain spontaneous or induced mutations leading to motor neuron death (Table 1). More recently, the mutated genes that lead to this phenotype were discovered. In addition and in an attempt to unravel the pathogenic mechanism(s) leading to ALS, a number of rodent models have been created starting from information on the genetic causes of ALS (Tables 2 and 3). Moreover, some transgenic animals show motor neuron degeneration as a prominent and unexpected feature 
while the (over)expressed genes do not cause ALS (Table 3). In this paper, we will give a systematic overview of the most important rodent models that show motor neuron degeneration and discuss the value and limitations.

\section{Spontaneous or Induced Motor Neuron Degeneration in Mice}

2.1. Wobbler Mouse. The Wobbler mouse arose as the result of a spontaneous mutation at the "Institute of Animal Genetics" in Edinburgh. These mice have an unsteady gait with progressive weakness. The most characteristic abnormality found in the Wobbler mouse is the degeneration of nerve cells of the motor system in the brainstem and in the spinal cord. This results in progressive denervation of skeletal muscles, leading to muscle atrophy especially in the head, neck, and forelimbs while the hind limbs are less severely affected [19]. The course of the disease varies in different mice and is also dependent on the genetic background [19, $24,25]$. Usually after a period of rapid deterioration up to the third or fourth month of age, the progress of the disease slows down. Some affected mice live for more than a year in spite of muscle weakness and wasting. In other cases, the disease causes a more extensive and severe muscle weakness and is fatal by the third or fourth month [19].

Originally, the Wobbler mouse was considered as a good model of motor neuron degeneration. However, this mouse model does not develop a typical motor neuron disease. The degeneration of motor neurons and the onset of astrogliosis and microgliosis in the spinal cord seem to be preceded by a more widespread neurodegeneration [26]. In addition, the Wobbler mice also show impaired spermiogenesis [27].

The gene mutated in this autosomal recessive disease model is VPS54 encoding a subunit of the Golgi-associated retrograde protein (GARP) complex that is required for tethering and fusion of endosome-derived transport vesicles to the trans-Golgi network, and the mutation leads to an amino acid replacement (Q967L) in the C-terminal domain of the Vps54 protein [28] (Table 1).

\subsection{Nmd Mouse Model. The "Neuromuscular degeneration"} (Nmd) mouse contains a spontaneous autosomal recessive mutation that leads to neuromuscular degeneration [20]. These mice develop a progressive motor neuron disease in which motor neuron loss causes muscle atrophy. Homozygous Nmd mice become progressively paralyzed and rarely survive longer than 4 weeks after birth.

A mutation that creates a cryptic donor splice site was found in intron 4 of the gene encoding the immunoglobulin $\mu$-binding protein 2 (IGHMBP2) [47], that colocalizes with the RNA-processing machinery. The consequence of this mutation is that the majority of the Ighmbp2 transcripts are aberrantly spliced and that a truncated Ighmbp 2 protein is formed. This mouse model became highly relevant after the discovery of Ighmbp2 mutations in patients suffering from spinal muscular atrophy with respiratory distress (SMARD) type 1 [48]. SMARD is an autosomal recessive motor neuron disease that affects infants. Patients present with respiratory distress due to diaphragmatic paralysis and progressive muscle weakness with predominant distal lower limb muscle involvement (Table 1).

2.3. Pmn Mouse. The "Progressive motor neuronopathy" (Pmn) mutant mouse develops hindlimb paralysis and displays progressive degeneration of motor axons while the cell bodies of the motor neurons in the ventral spinal cord are unaffected [21]. The Pmn mice die in the early postnatal period (6-7 weeks) [21]. The pathology in the Pmn mouse is considered as a distal axonopathy, and as a consequence the Pmn mouse may represent a good animal model of hereditary motor neuropathies.

The underlying genetic cause of the disease in the Pmn mouse is a point mutation in the TBCE gene on chromosome 13 [49]. The gene product is the tubulin-specific chaperone $\mathrm{E}$ (Tbce) that has an effect on microtubule stability and/or on the polymerization dynamics of microtubules. In humans, a deletion in Tbce is responsible for hypoparathyroidismretardation dysmorphism syndrome (HRD or Sanjad-Sakati syndrome, SSS) or autosomal recessive Kenny-Caffey syndrome (AR-KCS). Although neurological signs are part of the HRD/SSS phenotype, this syndrome is clinically variable, involves multiple tissues, and is quite different from the disease observed in the Pmn mouse (Table 1).

2.4. Loa and Cra Mice. Two independent N-ethyl-Nnitrosourea-(ENU) induced mutagenesis experiments generated the mutant mouse lines: "Legs at odd angles" (Loa) [22] and "Cramping 1" (Cra) [23]. These mice manifest progressive motor neuron disorders and show remarkable similarities to specific features of human pathology, including Lewy body-like inclusions containing SOD1, neurofilaments (NFs), and ubiquitin.

Two different point mutations in the gene encoding dynein were found in the Loa and Cra mice [68]. In the Loa mouse a mutation is present that changes phenylalanine to tyrosine at position 580 (F580Y), while the Cra mutation converts tyrosine 1055 to a cysteine (Y1055C). Dynein is a motor protein that is involved in retrograde transport and moves in the minus-end direction along microtubules. The genetic cause and phenotype of the Loa and Cra mouse models were often used as an argument in favor of the hypothesis that disturbances in axonal transport lead to selective motor neuron loss.

However, the validity of the Loa mouse as a model for ALS was questioned by the observation that no selective loss of motor neurons was detected [69]. In contrast, postnatal loss of lumbar proprioceptive sensory axons, accompanied by decreased excitatory glutamatergic input to motor neurons, was observed [69]. It was suggested that this reduction in glutamatergic input from lost proprioceptive sensory axons is the underlying cause of the unexpected prolongation of the life span of one of the mutant SOD1 mouse models (see below) after crossbreeding with the Loa mice [70]. This fits with the idea that excitotoxicity plays an important role in the selective motor neuron death underlying ALS [71].

Also the Cra mice do not show motor neuron loss, not even in aged animals [72]. In contrast, Cra mice 
TABLE 1: Overview of spontaneous or induced mouse models showing motor neuron degeneration.

\begin{tabular}{lcllll}
\hline Name & Mutated gene & Gene product & Inheritance & Human disease & Reference \\
\hline Wobbler & VPS54 & Subunit of the GARP complex & recessive & NA & {$[19]$} \\
Nmd & IGHMBP2 & Immunoglobulin $\mu$-binding protein 2 & recessive & SMARD1 & [20] \\
Pmn & TBCE & tubulin-specific chaperone E & recessive & motor neuropathy HRD/SSS & {$[21]$} \\
Loa & DYNC1H1 & dynactin & dominant & sensory neuropathy \\
Cra & DYNC1H1 & dynactin & dominant & sensory neuropathy & {$[22]$} \\
\hline
\end{tabular}

SMARD: spinal muscular atrophy with respiratory distress, HRD: hypoparathyroidism-retardation dysmorphism syndrome, SSS: Sanjad-Sakati syndrome, and NA: not available.

TABLE 2: Overview of transgenic rodent models for motor neuron degeneration (Mendelian and typical FALS).

\begin{tabular}{|c|c|c|c|c|c|}
\hline Disease & Gene product & Inheritance & Animal & Genetic modification & Reference \\
\hline \multirow[t]{13}{*}{ ALS1 } & Superoxide dismutase 1 & Dominant & Mouse & genomic hSOD1 G37R & [29] \\
\hline & & & & genomic hSOD1 G85R & {$[30]$} \\
\hline & & & & genomic mSOD1 G86R & {$[31]$} \\
\hline & & & & genomic hSOD1 G93A & {$[32]$} \\
\hline & & & & genomic hSOD1 L126Z(stop) & {$[33]$} \\
\hline & & & & genomic hSOD1 L126delTT & {$[34]$} \\
\hline & & & & genomic hSOD1 Quad & {$[35]$} \\
\hline & & & & PrP; cDNA SOD1 G37R & {$[35]$} \\
\hline & & & & Thy-1; cDNA hSOD1 G93A & {$[36]$} \\
\hline & & & Rat & genomic hSOD1 H46R & {$[37]$} \\
\hline & & & & genomic hSOD1 G93A & {$[37]$} \\
\hline & & & & genomic hSOD1 G93A & {$[38]$} \\
\hline & & Dominant/recessive & Mouse & genomic hSOD1 D90A & [39] \\
\hline ALS6 & FUS/TLS & Dominant (recessive) & Mouse & FUS/TLS KO & {$[40,41]$} \\
\hline \multirow[t]{7}{*}{ ALS10 } & TDP-43 & Dominant & Mouse & Prp; hTDP-43 A315T & [42] \\
\hline & & & & Thy-1; hTDP-43 WT & {$[43]$} \\
\hline & & & & PrP; hTDP-43 WT & {$[44]$} \\
\hline & & & & PrP; hTDP-43 A315T & {$[44]$} \\
\hline & & & & PrP; hTDP-43 M337V & {$[44]$} \\
\hline & & & & PrP; hTDP-43 WT & {$[45]$} \\
\hline & & & Rat & TRE; hTDP-43 M337V and WT & {$[46]$} \\
\hline
\end{tabular}

hSOD1: human superoxide dismutase 1, mSOD1: mouse superoxide dismutase 1, FUS: fused in sarcoma, TLS: translocated in liposarcoma, KO: knockout, PrP: prion promoter, TRE: tTA-activated promoter.

show a prominent degeneration of sensory neurons. As a consequence, it was suggested that the Cra mice should be considered as a model for sensory neuropathies rather than for motor neuron disease (Table 1). In addition, a recent study showed that the Cra mice also loose striatal neurons supporting a role for dynein dysfunction in the pathogenesis of neurodegenerative disorders of the basal ganglia such as Perry syndrome and Huntington's disease [73].

\section{Rodent Models of Typical Mendelian FALS}

\subsection{ALS1: Superoxide Dismutase 1; 21q}

3.1.1. Transgenic Mice. After the discovery of mutations in the SOD1 gene, a transgenic mouse overexpressing mutant (G93A) SOD1 was created by insertion of multiple copies of human genomic SOD1 into the mouse genome [32]. These transgenic mice show progressive hind limb weakness leading to paralysis and death and replicate in an almost perfect way the disease process in patients. The moment of disease onset and the life span of these mice are related to the level of overexpression of mutant SOD1, while overexpression of nonmutated SOD1 gives no phenotype.

In addition to the original mutant (G93A) SOD1 mice, a large number of other transgenic mice that (over)express human SOD1 containing other mutations (G37R, G85R or D90A) or mutant (G86R) mouse SOD1 were created (Table 2), and these mice show a similar phenotype as the mutant (G93A) SOD1 mouse line [29-31, 39]. Also transgenic mice overexpressing an SOD1 that was truncated at the C-terminus show clear motor neuron degeneration $[33,34]$.

The different mutant SOD1 mouse models have been extensively studied and strongly indicate that mutant SOD1 causes selective motor neuron death by a "gain of function" Moreover, these mice were used to study the pathogenic 
TABLE 3: Overview of transgenic mouse models for motor neuron degeneration (atypical or rare FALS and candidate genes).

\begin{tabular}{|c|c|c|c|c|c|}
\hline Disease & Gene product & Inheritance & Animal & Genetic modification & Reference \\
\hline \multirow[t]{4}{*}{ ALS2 } & Alsin & Recessive & Mouse & KO (exon 3) & {$[50]$} \\
\hline & & & & $\mathrm{KO}$ (stop codon in exon 3 ) & {$[51]$} \\
\hline & & & & KO (exon 3 and 4$)$ & {$[52]$} \\
\hline & & & & $\mathrm{KO}($ exon 4$)$ & {$[53]$} \\
\hline ALS8 & VAPB & Dominant & Mouse & PrP; VAPB P56S & {$[54]$} \\
\hline \multirow[t]{2}{*}{ ALS } & Dynactin & Dominant & Mouse & Knock-in G59S p150 Glued & {$[55]$} \\
\hline & & & & Thy-1; G59 p150 Glued & {$[56,57]$} \\
\hline CMT2E/1F & Neurofilament-L & Dominant & Mouse & NF-L L394P & {$[58]$} \\
\hline NA & Peripherin & NA & Mouse & overexpression & {$[59]$} \\
\hline NA & Vascular endothelial growth factor & NA & Mouse & $\mathrm{VEGF}^{\delta / \delta}$ & {$[60]$} \\
\hline \multirow[t]{6}{*}{ FTDP-tau } & tau & Dominant & Mouse & 4R human tau & {$[61]$} \\
\hline & & & & R406W human tau & {$[62,63]$} \\
\hline & & & & P301L human tau & {$[64]$} \\
\hline & & & & G272V, P301S human tau & {$[65]$} \\
\hline & & & & V337M human tau & {$[66]$} \\
\hline & & & & P301S human tau & {$[67]$} \\
\hline
\end{tabular}

PrP: prion promoter, CMT: Charcot-Marie-Tooth, FTDP: frontotemporal dementia with parkinsonism, and NA: not available.

changes occurring during the disease process, and they were also crossbred with many other transgenic mice to get insight about the pathogenic mechanism(s) involved. From one of these crossbreedings, it was concluded that mutant SOD1mediated oxidative abnormalities are not the primary cause of mutant SOD1 toxicity. SOD1 is an enzyme that requires copper to catalyze the conversion of toxic superoxide radicals into hydrogen peroxide and oxygen. Copper plays a crucial role in the normal and/or aberrant enzymatic activity of the enzyme, and copper loading of SOD1 is performed by a specific copper chaperone (CCS). Crossbreeding of transgenic mutant (G93A) SOD1 mice with knockout mice lacking the CCS, does not influence the life span of mutant (G93A) SOD1 mice [74]. Another argument that oxidative stress induced by mutant SOD1 is not the initiating factor is provided by the transgenic mice overexpressing mutant SOD1 in which the histidines that bind copper are mutated (SOD1-Quad). Two of these mutations are known human mutations that cause ALS, and the SOD1-Quad mice develop age-dependent motor neuron loss despite the lack of copper binding by SOD1 [35].

The mutant SOD1 models were also used to clarify the contribution of different cell types in the disease process. Selective (over)expression of mutant SOD1 in motor neurons [75] or in glial cells [76] is not sufficient to induce a motor phenotype, indicating that an interplay between different cell types is needed to get motor neuron death. However, it is unclear whether mutant SOD1 is expressed to a sufficiently high level in these transgenic mice. Other studies reported that exclusive neuronal expression of mutant SOD1 can cause motor neuron degeneration $[36,77]$ and can also lead to paralysis [36]. The involvement of nonneuronal cell types in mutant SOD1-induced motor neuron death was proven by using a transgenic mouse that contains a floxed mutant (G37R) SOD1 gene. This gene can be excised in different cell types by the action of Cre recombinase. Selective reduction of the amount of mutant SOD1 in microglia and peripheral macrophages has a dramatic effect and delays significantly the progression of the disease [78]. Moreover, reduction of mutant SOD1 expression in astrocytes also affects disease progression. This selective silencing of mutant SOD1 expression in astrocytes is obtained by crossbreeding transgenic mice containing floxed mutant SOD1 with mice expressing Cre driven by the glial fibrillary acidic protein (GFAP) promoter [79]. In the mutant (G37R) SOD1, the disease progression is significantly slowed, and the effect on survival is comparable to that of the selective removal of mutant (G37R) SOD1 from postnatal motor neurons [78, 79]. In mutant (G85R) SOD1 mice, the onset as well as the early disease phase is affected indicating that different mutant SOD1s may have different characteristics [80]. Altogether, these data show that the expression of mutant SOD1 in astrocytes and microglial cells has a negative effect on the disease process. The complexity of the mutant SOD1 mouse model is illustrated by the fact that selective removal of mutant SOD1 in Schwann cells has a negative effect on survival [81], while controversy exists on the role of (mutant) SOD1 in muscle cells [82-84].

The mutant SOD1 mouse models were also extensively used to test new therapeutic strategies. One famous example is minocycline, a drug that inhibits microglial activation and that shows a positive effect in three independent studies using two different mutant SOD1 models [85-87]. However, the subsequent clinical study was negative [88]. Because of this and many other failures, the validity of the mutant SOD1 models were questioned [89]. However, there could be many good reasons for the negative human clinical trials. First of all, the differences in pharmacokinetics between mice and humans are often largely neglected. In addition, it could be that the effects observed in the mouse are small and 
can be missed in a clinically and genetically heterogeneous human ALS population. One also has to realize that some positive results in mice were obtained from underpowered studies in which the treatment is started before disease onset $[89,90]$, while human clinical trials are done with patients already suffering from ALS. In order to circumvent these problems, clear guidelines were formulated outlining the standard operating procedures (SOPs) for preclinical animal research [91]. Last but not least, it is also possible that mutant SOD1 mice do not model sporadic ALS, but only represent an animal model of familial ALS or even only of familial ALS caused by mutations in SOD1 [89].

3.1.2. Transgenic Rats. Transgenic rats overexpressing mutant (H46R and G93A) SOD1 were also created and these animals also develop an age-dependent degeneration of motor neurons leading to paralysis and death [37, 38]. The advantage of this larger rat model is that it allows studies involving complex manipulations of the spinal fluid and spinal cord (e.g., implantation of intrathecal catheters for chronic therapeutic studies, sampling of cerebrospinal fluid,...). A major difference between the rat and mouse mutant SOD1 models is the more rapid progression of the disease in rats which could be due to respiratory insufficiency resulting from the degeneration of respiratory motor neurons [92]. In addition, part of the transgenic rats show weakness in the forelimbs as first clinical sign [38], a phenotype that becomes more pronounced after chronic stimulation of the phrenic nerve [93].

3.2. ALS6: FUS/TLS; 16p11. Recently, two studies reported the discovery of mutations in FUS (fused in sarcoma)/TLS (translocated in liposarcoma) as a new genetic cause of ALS in Cape Verde, the USA, Australia and the UK $[10,11]$. The FUS/TLS gene is located in a region on chromosome 16 previously associated with ALS6 [94]. FUS/TLS mutations were also found in other populations in Europe and the US and it is estimated that FUS/TLS mutations are the cause of familial ALS in $4 \%-5 \%$ of cases. FUS/TLS linked ALS is a dominant disease, except in the original Cape Verdean family in which the FUS mutation is recessive [10]. Although in-frame deletions and insertions have been reported, most mutations are missense, and the majority is located in the last exon.

The FUS/TLS protein is involved in RNA metabolism and regulation of transcription. FUS/TLS knockout mice die immediately after birth [40] or are rarely alive at weaning [41] (Table 2). In an outbred strain, FUS/TLS knock out mice survive but show male sterility and reduced fertility of females [41]. Neurons deficient for FUS/TLS show abnormal spine morphology and lower spine density [95]. No loss of motor neurons and/or a motor phenotype were reported indicating that mutant FUS/TLS induces the disease by a "gain-of-function" mechanism. Currently, no transgenic mice and/or rats are available that (over)express mutant and/or wild type FUS/TLS.

3.3. ALS10: TDP-43; $1 p 36$. Transactivation response DNAbinding protein with molecular weight $43 \mathrm{kD}$ (TDP-43) was first identified as one of the major constituents of the intraneuronal inclusions observed in ALS and frontotemporal lobar degeneration with ubiquitin-positive inclusions (FTLD-U) [96]. Subsequently, mutations in the TARDBP gene encoding TDP-43 were identified in patients with familial ALS [5-9]. The mutations found in the TARDBP gene are missense mutations and are almost exclusively located in the C-terminal part of the protein [97]. TARDBP mutations are rare and account for less than $5 \%$ of familial ALS.

TDP-43 has two RNA binding domains and a glycinerich domain in the C-terminal part, with which it binds to various heterogeneous nuclear nucleoproteins (hnRNPs). It is more abundantly present in the nucleus than in the cytoplasm. Although the exact role of TDP-43 is incompletely understood, it is thought to play a role in RNA processing, stabilisation, and transport $[98,99]$.

Transgenic mice were created by overexpression of mutant (A315T) TDP-43 under the control of the mouse prion promoter [42]. These transgenic mice are born at normal Mendelian ratios, have the same weight as nontransgenic littermates, and appear normal up to 3 months of age [42]. From that moment on, the transgenic mice develop gait abnormalities, begin to lose weight, and develop a "swimming" gait. The life span of the animals is approximately 5 months. These transgenic animals show degeneration of motor neurons and of layer 5 pyramidal neurons in the frontal cortex. The degenerating neurons contain ubiquitinated aggregates, negative for (mutant) TDP-43. The authors were not able to create wild-type TDP43 overexpressing mice and as a consequence the question remained whether these findings are the result of the TDP-43 mutation or of the overexpression of TDP-43.

The latter seems to be the case as overexpression of human wild-type TDP-43 driven by the Thy-1 promotor also results in toxicity. These transgenic mice develop gait abnormalities and show degeneration of motor neurons and neurons in layer 5 of the frontal cortex [43]. In addition, nuclear and cytoplasmic aggregates of ubiquitinated and phosphorylated TDP-43 are clearly present [43]. A number of recent studies confirm these findings in transgenic mice that overexpress wild-type TDP-43 under the control of the prion promoter $[44,45]$ (Table 2). Altogether, these data indicate that it is the overexpression of TDP-43 that is toxic.

In addition to the different mouse models, a conditional rat model was created in which wild-type TDP-43 or mutant $(\mathrm{M} 337 \mathrm{~V})$ TDP-43 is expressed under the control of a promoter that can be silenced by treating the transgenic rats with doxycycline [46] (Table 2). While constitutive or early embryonic expression of the mutant (M337V) TDP43 transgene causes embryonic death, postnatal expression of the transgene induces paralysis and death. The severity of the phenotype differs between the different lines and relates to the copy number of the transgene. In contrast, wild-type TDP-43 overexpression does not lead to a disease phenotype despite the fact that the transgene is expressed at a comparable level, indicating that the phenotype is due to mutations in TDP-43 and not to overexpression of TDP-43 [46]. The mutant (M337V) TDP-43 transgenic rats 
show neuronal degeneration, initially of motor neurons but in a later disease state also of cortical, hippocampal, and cerebellar neurons [46]. Also in the rat model, ubiquitinated TDP-43 inclusions are scarce indicating that this rat model does not replicate all the pathogenic features observed in ALS patients. Most likely, a more sophisticated approach such as a "knock-out/knock-in" mouse will be necessary to solve this problem and to obtain a good mutant TDP-43 related rodent model of ALS.

\section{Rodent Models of Atypical or Rare Mendelian FALS}

4.1. ALS2: Alsin; 2q33. ALS2 is a rare autosomal, recessive form of ALS that is characterized by a juvenile onset of progressive spasticity in the limbs, facial, and pharyngeal muscles. In families of Arabic origin, mutations in the ALS2 gene on chromosome 2 were discovered [12]. At present, at least 11 different mutations in the ALS2 gene were reported leading either to ALS, or to primary lateral sclerosis (PLS), or to infantile onset ascending hereditary spastic paraplegia (IAHSP) with only upper motor neurons involved (for a review see [1]). All patients are homozygous for the mutant gene, and all reported mutations lead to premature termination of the transcript and as a consequence to a truncated protein.

Several groups generated an alsin knockout mouse [5053] (Table 3), but these mice had no clear motor phenotype. Primary motor neuron cultures from the first line of alsin knockout mice are more susceptible to oxidative stress [50]. The second transgenic mouse model shows an agedependent, slowly progressive loss of cerebellar Purkinje cells and a disturbance of spinal motor neurons associated with astrocytosis and microglial cell activation, indicating a subclinical dysfunction of the motor system [51]. The third mouse model shows a mild, age-independent hypoactivity and smaller cortical motor neurons. In addition, disturbances in endosomal transport were reported [52]. The fourth transgenic model shows slowed movement without muscle weakness and progressive axonal degeneration in the lateral spinal cord [53]. From all these observations, it can be concluded that knocking out alsin in mouse is insufficient to cause major motor deficits, while subtle behavioral changes and a clear loss in cerebellar Purkinje cells are present.

4.2. ALS8: VAPB; 20q13. A missense mutation in the VAPB gene was found in a Brazilian family [15], and this mutation results in different clinical manifestations including lateonset spinal muscular atrophy and late-onset atypical ALS with slow progression. To date, there are 8 families of which 7 are of Portuguese-Brazilian and one is of AfricanBrazilian origin. Both wild-type and mutant VAPB, were overexpressed in neurons. Recently, transgenic mice were created that express either wild-type VAPB or mutant (P56S) VAPB in the nervous system using a modified prion promoter [54]. These mice develop no overt motor phenotype and show no alterations in survival. Interestingly, mutant (P56S) VAPB overexpressing mice show cytoplasmic TDP-43 accumulations in the motor neurons of the spinal cord [54].
4.3. Dynactin; 2p13. A G59S mutation located in the microtubule-binding domain of dynactin p150 Glued was described as the cause of an autosomal dominant, lateonset motor neuron disease in a large family of European descent [18]. Initial in vitro microtubule binding studies indicated that mutant $\mathrm{p} 150^{\text {Glued }}$ exhibit a reduced binding efficiency to microtubules [18], which is consistent with a "loss-of-function." In addition, cell culture experiments demonstrated that mutant G59S p150 Glued perturbs dynactin function and causes protein aggregation [100]. More recently, a number of mouse models were created from which it can be concluded that motor neuron loss is caused by a dominant negative mechanism $[55,56]$. Lai et al. (2007) generated a mutant G59S p150 Glued knock-in mouse. Early embryonic lethality is observed in the mutant G59S p150 Glued homozygous knock-in mice, while the heterozygous mutant G59S p 150 Glued mice start to display a motor neuron diseaselike phenotype at 10 months of age. This is accompanied by excessive accumulation of cytoskeletal and synaptic vesicle proteins at the neuromuscular junctions, loss of spinal motor neurons, increase of astrogliosis, and shortening of gait [55]. Laird et al. (2008) and Chevalier-Larsen et al. (2008) constructed transgenic mice that overexpress mutant G59S p150 Glued under the control of the neuron-specific Thy-1 promoter $[56,57]$. These mice also display a motor phenotype resulting in muscle weakness, paralysis, and/or death (Table 3 ).

\section{Rodent Models Based on Candidate Genes}

5.1. Neurofilaments and Peripherin. Neurofilaments (NFs) are the most abundant intermediate filaments in neurons and consist of three subunits: NF-L, NF-M, and NF-H. NF accumulations are found in both familial and sporadic ALS cases [101, 102]. In a limited number of ALS patients, mutations in the KSP phosphorylation domain seem to be present [103]. Over the years, a large number of transgenic mice with modifications related to NFs were made in an attempt to determine the role of these intermediate filaments in the pathology of motor neurons (for a review see [104]).

Knockout mice for these different subunits alone or double transgenic mice deficient in two NF subunits do not show a clear phenotype, although some of these mice show a loss of motor axons. Also overexpression of the different NF subunits does not induce motor neuron death. In some of these transgenic mice NF accumulations in neuronal cell bodies were found, but this does not induce motor neuron death. However, NF abnormalities can induce selective motor neuron death in vivo as indicated by a transgenic mouse expressing a mutant NF-L. In these mice, leucine at position 394 was mutated into a proline, and this causes a dominant motor neuron disease [58]. These transgenic mice show massive, selective degeneration of motor neurons accompanied by accumulations of NFs, although no effect on the life span of these mice was reported. This mouse model is very interesting and relevant as NF-L mutations are the cause of a dominantly inherited motor neuropathy: CharcotMarie-Tooth disease, type 2E [105]. 
More recently and because of the proposed role of RNA metabolism in the pathogenesis of ALS, a transgenic mouse overexpressing the $3^{\prime}$-UTR of NF-L received renewed interest [106]. This transgenic mouse presents with neuronal aggregates, astrocytosis, and impaired motor function [107]. As the endogenous level of NF-L protein is normal, it is suggested that sequences in the $3^{\prime}$-UTR of NF-L bind transacting factors that are essential for a normal motor neuron function [106].

A frameshift deletion and a mutation in the peripherin gene encoding another intermediate filament protein were discovered in ALS patients $[108,109]$. Peripherin is mostly expressed in the peripheral nervous system and is upregulated both in the peripheral and central nervous system after injury and by inflammatory cytokines. Transgenic mice that overexpress wild-type peripherin develop motor dysfunctions very late in their life (after 2 years). This phenotype is accompanied by the loss of motor axons and by the appearance of peripherin inclusion bodies in the motor neurons. The onset of motor dysfunction and axonal loss is dramatically accelerated by the absence of NF-L, as revealed by crossbreeding of peripherin overexpressing mice with NF$\mathrm{L}$ knockout mice. These double transgenic mice also show a dramatic loss of motor neurons [59].

5.2. Growth Factors. Survival of motor neurons is dependent on the presence of growth factors. Absence of these neurotrophic factors can cause motor neuron death, as is illustrated by the phenotype of the transgenic mice in which the gene for ciliary neurotrophic factor (CNTF) is deleted [110]. CNTF is a cytosolic protein, expressed at high levels in myelinating Schwann cells, promoting survival of motor neurons in vitro. These knockout mice have no phenotype during the postnatal weeks, but develop atrophy and loss of motor neurons with aging [110].

Another example of motor neuron death induced by insufficient growth factors is the $\mathrm{VEGF}^{\delta / \delta}$ transgenic mouse. This mouse model was created by deleting the hypoxiaresponse element (HRE) in the promoter region of the gene encoding VEGF. This modification induces an adult-onset, slowly progressive motor neuron loss leading to muscle atrophy and a motor phenotype [60]. Despite the fact that the $\mathrm{VEGF}^{\delta / \delta}$ mice show clear motor neuron loss and muscle atrophy, these animals do not become paralyzed and have a normal life span.

5.3. Tau. Manipulations involving the gene encoding the microtubule-associated protein, tau, also resulted in a number of mouse models showing a clear motor phenotype. Tau is an axonal phosphoprotein that establishes short crossbridges between axonal microtubules and, thereby, supports intracellular trafficking, including axonal transport. In neurons affected by a tauopathy, tau is hyperphosphorylated and is located not only in axons but also in cell bodies and dendrites. Tau is the major component of the intracellular filamentous deposits found in a number of neurodegenerative diseases including Alzheimer's disease, while mutations in tau are associated with frontotemporal dementia with parkinsonism (FTDP) (for a review see [111]). Several tau transgenic mice show a progressive motor phenotype with muscle atrophy. Overexpression of mutant tau also induces age-dependent accumulation of insoluble filamentous tau aggregates in neuronal perikarya of spinal cord, a motor phenotype and a reduced life span [61-67] (Table 3). Despite these motor phenotypes, evidence is lacking that tau plays a role in the pathogenesis of ALS [112].

\section{Conclusions}

For more than a decade, the mutant SOD1 mice and rats have been the prototype of an ideal model to study (selective) motor neuron death, the hallmark of ALS. However, the major frustration is that this almost perfect model did not lead to a major breakthrough on the therapeutic level. All positive therapeutic interventions in this model failed in subsequent human clinical trials. As a consequence, the ALS field is eagerly awaiting new rodent models that are generated starting from other mutated genes. Until recently, these attempts were based on genetic defects causing atypical or very rare forms of familial ALS. Since the discovery of mutations in the genes encoding TDP-43 and FUS/TLS, hope arises that (over) expression of these mutated genes will lead to the generation of new ALS models. Despite a large number of attempts, the rodent models based on (mutant) TDP-43 are not yet perfect. These models lack specificity in relation to the mutation and/or the cell type affected. The value of the rodent models that are the result of modification of genes that unexpectedly cause a motor neuron phenotype is unclear. In the best case, these models point to a new pathogenic mechanism. None of these models is currently used for routine drug screening.

In conclusion, the mutant SOD1 rodent models remains the best models currently available to study the pathogenesis of ALS and to test new therapies, although the question remains how representative this model is for human sporadic and familial ALS. As a consequence, searching for more and better rodent models based on mutations in new genes remains an important challenge in the ALS field.

\section{Acknowledgments}

Research of the author is supported by grants from the "Fund for Scientific Research Flanders" (FWO-Vlaanderen), the University of Leuven, the Belgian Government (Interuniversity Attraction Poles, programme P6/43 of the Belgian Federal Science Policy Office), the "Association Belge contre les Maladies neuro-Musculaires" (ABMM), the "Association Française contre les Myopathies" (AFM), the "Frick Foundation for ALS Research" and the "Fondation Thierry Latran."

\section{References}

[1] F. Gros-Louis, C. Gaspar, and G. A. Rouleau, "Genetics of familial and sporadic amyotrophic lateral sclerosis," Biochimica et Biophysica Acta, vol. 1762, no. 11-12, pp. 956-972, 2006.

[2] P. A. Dion, H. Daoud, and G. A. Rouleau, "Genetics of motor neuron disorders: new insights into pathogenic 
mechanisms," Nature Reviews Genetics, vol. 10, no. 11, pp. 769-782, 2009.

[3] L. Van Den Bosch and V. Timmerman, "Genetics of motor neuron disease," Current Neurology and Neuroscience Reports, vol. 6, no. 5, pp. 423-431, 2006.

[4] D. R. Rosen, T. Siddique, D. Patterson et al., "Mutations in $\mathrm{Cu} / \mathrm{Zn}$ superoxide dismutase gene are associated with familial amyotrophic lateral sclerosis," Nature, vol. 362, no. 6415, pp. 59-62, 1993.

[5] J. Sreedharan, I. P. Blair, V. B. Tripathi et al., "TDP43 mutations in familial and sporadic amyotrophic lateral sclerosis," Science, vol. 319, no. 5870, pp. 1668-1672, 2008.

[6] E. Kabashi, P. N. Valdmanis, P. Dion et al., "TARDBP mutations in individuals with sporadic and familial amyotrophic lateral sclerosis," Nature Genetics, vol. 40, no. 5, pp. 572-574, 2008.

[7] V. M. Van Deerlin, J. B. Leverenz, L. M. Bekris et al., "TARDBP mutations in amyotrophic lateral sclerosis with TDP-43 neuropathology: a genetic and histopathological analysis," The Lancet Neurology, vol. 7, no. 5, pp. 409-416, 2008.

[8] M. A. Gitcho, R. H. Baloh, S. Chakraverty et al., "TDP-43 A315T mutation in familial motor neuron disease," Annals of Neurology, vol. 63, no. 4, pp. 535-538, 2008.

[9] A. Yokoseki, A. Shiga, C. F. Tan et al., "TDP-43 mutation in familial amyotrophic lateral sclerosis," Annals of Neurology, vol. 63, no. 4, pp. 538-542, 2008.

[10] T. J. Kwiatkowski, D. A. Bosco, A. L. LeClerc et al., "Mutations in the FUS/TLS gene on chromosome 16 cause familial amyotrophic lateral sclerosis," Science, vol. 323, no. 5918, pp. 1205-1208, 2009.

[11] C. Vance, B. Rogelj, T. Hortobágyi et al., "Mutations in FUS, an RNA processing protein, cause familial amyotrophic lateral sclerosis type 6," Science, vol. 323, no. 5918, pp. 1208 1211, 2009.

[12] S. Hadano, C. K. Hand, H. Osuga et al., "A gene encoding a putative GTPase regulator is mutated in familial amyotrophic lateral sclerosis 2," Nature Genetics, vol. 29, no. 2, pp. 166173, 2001.

[13] Y. Z. Chen, C. L. Bennett, H. M. Huynh et al., "DNA/RNA helicase gene mutations in a form of juvenile amyotrophic lateral sclerosis (ALS4)," American Journal of Human Genetics, vol. 74, no. 6, pp. 1128-1135, 2004.

[14] A. Orlacchio, C. Babalini, A. Borreca et al., "SPATACSIN mutations cause autosomal recessive juvenile amyotrophic lateral sclerosis," Brain, vol. 133, no. 2, pp. 591-598, 2010.

[15] A. L. Nishimura, M. Mitne-Neto, H. C. A. Silva et al., "A mutation in the vesicle-trafficking protein VAPB causes late-onset spinal muscular atrophy and amyotrophic lateral sclerosis," American Journal of Human Genetics, vol. 75, no. 5, pp. 822-831, 2004.

[16] M. J. Greenway, P. M. Andersen, G. Russ et al., "ANG mutations segregate with familial and 'sporadic' amyotrophic lateral sclerosis," Nature Genetics, vol. 38, no. 4, pp. 411-413, 2006.

[17] H. Maruyama, H. Morino, H. Ito et al., "Mutations of optineurin in amyotrophic lateral sclerosis," Nature, vol. 465, no. 7295, pp. 223-226, 2010.

[18] I. Puls, C. Jonnakuty, B. H. LaMonte et al., "Mutant dynactin in motor neuron disease," Nature Genetics, vol. 33, no. 4, pp. 455-456, 2003.

[19] L. W. Duchen and S. J. Strich, "An hereditary motor neurone disease with progressive denervation of muscle in the mouse: the mutant 'wobbler,' Journal of Neurology Neurosurgery and Psychiatry, vol. 31, no. 6, pp. 535-542, 1968.

[20] S. A. Cook, K. R. Johnson, R. T. Bronson, and M. T. Davisson, "Neuromuscular degeneration (nmd): a mutation on mouse chromosome 19 that causes motor neuron degeneration," Mammalian Genome, vol. 6, no. 3, pp. 187-191, 1995.

[21] H. Schmalbruch, H. J. S. Jensen, M. Bjaerg, Z. Kamieniecka, and L. Kurland, "A new mouse mutant with progressive motor neuronopathy," Journal of Neuropathology and Experimental Neurology, vol. 50, no. 3, pp. 192-204, 1991.

[22] D. C. Rogers, JO. Peters, J. E. Martin et al., "SHIRPA, a protocol for behavioral assessment: validation for longitudinal study of neurological dysfunction in mice," Neuroscience Letters, vol. 306, no. 1-2, pp. 89-92, 2001.

[23] M. H. De Angelis, H. Flaswinkel, H. Fuchs et al., "Genomewide, large-scale production of mutant mice by ENU mutagenesis," Nature Genetics, vol. 25, no. 4, pp. 444-447, 2000.

[24] M. Ulbrich, V. C. Schmidt, M. Ronsiek et al., "Genetic modifiers that aggravate the neurological phenotype of the wobbler mouse," NeuroReport, vol. 13, no. 4, pp. 535-539, 2002.

[25] K. Kaupmann, D. Simon-Chazottes, J. L. Guenet, and H. Jockusch, "Wobbler, a mutation affecting motoneuron survival and gonadal functions in the mouse, maps to proximal chromosome 11," Genomics, vol. 13, no. 1, pp. 39-43, 1992.

[26] S. Rathke-Hartlieb, V. C. Schmidt, H. Jockusch, T. SchmittJohn, and J. W. Bartsch, "Spatiotemporal progression of neurodegeneration and glia activation in the wobbler neuropathy of the mouse," NeuroReport, vol. 10, no. 16, pp. 3411-3416, 1999.

[27] P. Heimann, S. Laage, and H. Jockusch, "Defect of sperm assembly in a neurological mutant of the mouse, wobbler (WR)," Differentiation, vol. 47, no. 2, pp. 77-83, 1991.

[28] T. Schmitt-John, C. Drepper, A. Mußmann et al., "Mutation of Vps54 causes motor neuron disease and defective spermiogenesis in the wobbler mouse," Nature Genetics, vol. 37, no. 11, pp. 1213-1215, 2005.

[29] P. C. Wong, C. A. Pardo, D. R. Borchelt et al., "An adverse property of a familial ALS-linked SOD1 mutation causes motor neuron disease characterized by vacuolar degeneration of mitochondria," Neuron, vol. 14, no. 6, pp. 1105-1116, 1995.

[30] L. I. Bruijn, M. W. Becher, M. K. Lee et al., "ALS-linked SOD1 mutant G85R mediates damage to astrocytes and promotes rapidly progressive disease with SOD1-containing inclusions," Neuron, vol. 18, no. 2, pp. 327-338, 1997.

[31] M. E. Ripps, G. W. Huntley, P. R. Hof, J. H. Morrison, and J. W. Gordon, "Transgenic mice expressing an altered murine superoxide dismutase gene provide an animal model of amyotrophic lateral sclerosis," Proceedings of the National Academy of Sciences of the United States of America, vol. 92, no. 3, pp. 689-693, 1995.

[32] M. E. Gurney, H. Pu, A. Y. Chiu et al., "Motor neuron degeneration in mice that express a human $\mathrm{Cu}, \mathrm{Zn}$ superoxide dismutase mutation," Science, vol. 264, no. 5166, pp. 17721775, 1994.

[33] J. Wang, G. Xu, H. Li et al., "Somatodendritic accumulation of misfolded SOD1-L126Z in motor neurons mediates degeneration: $\alpha \mathrm{B}$-crystallin modulates aggregation," Human Molecular Genetics, vol. 14, no. 16, pp. 2335-2347, 2005.

[34] Y. Watanabe, K. Yasui, T. Nakano et al., "Mouse motor neuron disease caused by truncated SOD1 with or without C-terminal modification," Molecular Brain Research, vol. 135, no. 1-2, pp. 12-20, 2005. 
[35] J. Wang, H. Slunt, V. Gonzales et al., "Copper-binding-sitenull SOD1 causes ALS in transgenic mice: aggregates of nonnative SOD1 delineate a common feature," Human Molecular Genetics, vol. 12, no. 21, pp. 2753-2764, 2003.

[36] D. Jaarsma, E. Teuling, E. D. Haasdijk, C. I. De Zeeuw, and C. C. Hoogenraad, "Neuron-specific expression of mutant superoxide dismutase is sufficient to induce amyotrophic lateral sclerosis in transgenic mice," Journal of Neuroscience, vol. 28, no. 9, pp. 2075-2088, 2008.

[37] M. Nagai, M. Aoki, I. Miyoshi et al., "Rats expressing human cytosolic copper-zinc superoxide dismutase transgenes with amyotrophic lateral sclerosis: associated mutations develop motor neuron disease," Journal of Neuroscience, vol. 21, no. 23, pp. 9246-9254, 2001.

[38] D. S. Howland, J. Liu, Y. She et al., "Focal loss of the glutamate transporter EAAT2 in a transgenic rat model of SOD1 mutant-mediated amyotrophic lateral sclerosis (ALS)," Proceedings of the National Academy of Sciences of the United States of America, vol. 99, no. 3, pp. 1604-1609, 2002.

[39] P. A. Jonsson, K. S. Graffmo, T. Brännström, P. Nilsson, P. M. Andersen, and S. L. Marklund, "Motor neuron disease in mice expressing the wild type-like D90A mutant superoxide dismutase-1," Journal of Neuropathology and Experimental Neurology, vol. 65, no. 12, pp. 1126-1136, 2006.

[40] G. G. Hicks, N. Singh, A. Nashabi et al., "Fus deficiency in mice results in defective B-lymphocyte development and activation, high levels of chromosomal instability and perinatal death," Nature Genetics, vol. 24, no. 2, pp. 175-179, 2000.

[41] M. Kuroda, J. Sok, L. Webb et al., "Male sterility and enhanced radiation sensitivity in TLS(-/-) mice," EMBO Journal, vol. 19, no. 3, pp. 453-462, 2000.

[42] I. Wegorzewska, S. Bell, N. J. Cairns, T. M. Miller, and R. H. Baloh, "TDP-43 mutant transgenic mice develop features of ALS and frontotemporal lobar degeneration," Proceedings of the National Academy of Sciences of the United States of America, vol. 106, no. 44, pp. 18809-18814, 2009.

[43] H. Wils, G. Kleinberger, J. Janssens et al., “TDP-43 transgenic mice develop spastic paralysis and neuronal inclusions characteristic of ALS and frontotemporal lobar degeneration," Proceedings of the National Academy of Sciences of the United States of America, vol. 107, no. 8, pp. 3858-3863, 2010.

[44] N. R. Stallings, K. Puttaparthi, C. M. Luther, D. K. Burns, and J. L. Elliott, "Progressive motor weakness in transgenic mice expressing human TDP-43," Neurobiology of Disease, vol. 40, no. 2, pp. 404-414, 2010.

[45] Y.-F. Xu, T. F. Gendron, Y.-J. Zhang et al., "Wild-type human TDP-43 expression causes TDP-43 phosphorylation, mitochondrial aggregation, motor deficits, and early mortality in transgenic mice," Journal of Neuroscience, vol. 30, no. 32, pp. 10851-10859, 2010.

[46] H. Zhou, C. Huang, H. Chen et al., "Transgenic rat model of neurodegeneration caused by mutation in the TDP gene," PLoS Genetics, vol. 6, no. 3, Article ID e1000887, 2010.

[47] G. A. Cox, C. L. Mahaffey, and W. N. Frankel, "Identification of the mouse neuromuscular degeneration gene and mapping of a second site suppressor allele," Neuron, vol. 21, no. 6, pp. 1327-1337, 1998.

[48] K. Grohmann, M. Schuelke, A. Diers et al., "Mutations in the gene encoding immunoglobulin $\mu$-binding protein 2 cause spinal muscular atrophy with respiratory distress type 1 ," Nature Genetics, vol. 29, no. 1, pp. 75-77, 2001.
[49] N. Martin, J. Jaubert, P. Gounon et al., "A missense mutation in Tbce causes progressive motor neuronopathy in mice," Nature Genetics, vol. 32, no. 3, pp. 443-447, 2002.

[50] H. Cai, X. Lin, C. Xie et al., "Loss of ALS2 function is insufficient to trigger motor neuron degeneration in knockout mice but predisposes neurons to oxidative stress," Journal of Neuroscience, vol. 25, no. 33, pp. 7567-7574, 2005.

[51] S. Hadano, S. C. Benn, S. Kakuta et al., "Mice deficient in the Rab5 guanine nucleotide exchange factor ALS2/alsin exhibit age-dependent neurological deficits and altered endosome trafficking," Human Molecular Genetics, vol. 15, no. 2, pp. 233-250, 2006.

[52] R. S. Devon, P. C. Orban, K. Gerrow et al., "Als2-deficient mice exhibit disturbances in endosome trafficking associated with motor behavioral abnormalities," Proceedings of the National Academy of Sciences of the United States of America, vol. 103, no. 25, pp. 9595-9600, 2006.

[53] K. Yamanaka, T. M. Miller, M. McAlonis-Downes, S. J. Chun, and D. W. Cleveland, "Progressive spinal axonal degeneration and slowness in ALS2-deficient mice," Annals of Neurology, vol. 60, no. 1, pp. 95-104, 2006.

[54] E. L. Tudor, C. M. Galtrey, M. S. Perkinton et al., "Amyotrophic lateral sclerosis mutant vesicle-associated membrane protein-associated protein-B transgenic mice develop TAR-DNA-binding protein-43 pathology," Neuroscience, vol. 167, no. 3, pp. 774-785, 2010.

[55] C. Lai, X. Lin, J. Chandran, H. Shim, W. J. Yang, and H. Cai, "The G59S mutation in p150 causes dysfunction of dynactin in mice," Journal of Neuroscience, vol. 27, no. 51, pp. 1398213990, 2007.

[56] F. M. Laird, M. H. Farah, S. Ackerley et al., "Motor neuron disease occurring in a mutant dynactin mouse model is characterized by defects in vesicular trafficking," Journal of Neuroscience, vol. 28, no. 9, pp. 1997-2005, 2008.

[57] E. S. Chevalier-Larsen, K. E. Wallace, C. R. Pennise, and E. L. F. Holzbaur, "Lysosomal proliferation and distal degeneration in motor neurons expressing the G59S mutation in the p150 subunit of dynactin," Human Molecular Genetics, vol. 17, no. 13, pp. 1946-1955, 2008.

[58] M. K. Lee, J. R. Marszalek, and D. W. Cleveland, "A mutant neurofilament subunit causes massive, selective motor neuron death: implications for the pathogenesis of human motor neuron disease," Neuron, vol. 13, no. 4, pp. 975-988, 1994.

[59] J. M. Beaulieu, M. D. Nguyen, and J. P. Julien, "Late onset death of motor neurons in mice overexpressing wild-type peripherin," Journal of Cell Biology, vol. 147, no. 3, pp. 531544, 1999.

[60] B. Oosthuyse, L. Moons, E. Storkebaum et al., "Deletion of the hypoxia-response element in the vascular endothelial growth factor promoter causes motor neuron degeneration," Nature Genetics, vol. 28, no. 2, pp. 131-138, 2001.

[61] K. Spittaels, C. Van Den Haute, JO. Van Dorpe et al., "Prominent axonopathy in the brain and spinal cord of transgenic mice overexpressing four-repeat human tau protein," American Journal of Pathology, vol. 155, no. 6, pp. 2153-2165, 1999.

[62] B. Zhang, M. Higuchi, Y. Yoshiyama et al., "Retarded axonal transport of R406W mutant tau in transgenic mice with a neurodegenerative tauopathy," Journal of Neuroscience, vol. 24, no. 19, pp. 4657-4667, 2004.

[63] Y. Tatebayashi, T. Miyasaka, DE. H. Chui et al., “Tau filament formation and associative memory deficit in aged mice expressing mutant (R406W) human tau," Proceedings of the 
National Academy of Sciences of the United States of America, vol. 99, no. 21, pp. 13896-13901, 2002.

[64] M. Ramsden, L. Kotilinek, C. Forster et al., “Age-dependent neurofibrillary tangle formation, neuron loss, and memory impairment in a mouse model of human tauopathy (P301L)," Journal of Neuroscience, vol. 25, no. 46, pp. 1063710647, 2005.

[65] K. Schindowski, A. Bretteville, K. Leroy et al., "Alzheimer’s disease-like tau neuropathology leads to memory deficits and loss of functional synapses in a novel mutated tau transgenic mouse without any motor deficits," American Journal of Pathology, vol. 169, no. 2, pp. 599-616, 2006.

[66] K. Tanemura, M. Murayama, T. Akagi et al., "Neurodegeneration with tau accumulation in a transgenic mouse expressing V337M human tau," Journal of Neuroscience, vol. 22, no. 1, pp. 133-141, 2002.

[67] Y. Yoshiyama, M. Higuchi, B. Zhang et al., "Synapse Loss and Microglial Activation Precede Tangles in a P301S Tauopathy Mouse Model," Neuron, vol. 53, no. 3, pp. 337-351, 2007.

[68] M. Hafezparast, R. Klocke, C. Ruhrberg et al., "Mutations in dynein link motor neuron degeneration to defects in retrograde transport," Science, vol. 300, no. 5620, pp. $808-$ $812,2003$.

[69] H. S. Ilieva, K. Yamanaka, S. Malkmus et al., "Mutant dynein (Loa) triggers proprioceptive axon loss that extends survival only in the SOD1 ALS model with highest motor neuron death," Proceedings of the National Academy of Sciences of the United States of America, vol. 105, no. 34, pp. 12599-12604, 2008.

[70] D. Kieran, M. Hafezparast, S. Bohnert et al., "A mutation in dynein rescues axonal transport defects and extends the life span of ALS mice," Journal of Cell Biology, vol. 169, no. 4, pp. 561-567, 2005.

[71] L. Van Den Bosch, P. Van Damme, E. Bogaert, and W. Robberecht, "The role of excitotoxicity in the pathogenesis of amyotrophic lateral sclerosis," Biochimica et Biophysica Acta, vol. 1762, no. 11-12, pp. 1068-1082, 2006.

[72] L. Dupuis, A. Fergani, K. E. Braunstein et al., "Mice with a mutation in the dynein heavy chain 1 gene display sensory neuropathy but lack motor neuron disease," Experimental Neurology, vol. 215, no. 1, pp. 146-152, 2009.

[73] K. E. Braunstein, J. Eschbach, K. Ròna-Vörös et al., "A point mutation in the dynein heavy chain gene leads to striatal atrophy and compromises neurite outgrowth of striatal neurons," Human Molecular Genetics, vol. 19, no. 22, pp. 4385-4398, 2010.

[74] J. R. Subramaniam, W. E. Lyons, J. Liu et al., "Mutant SOD1 causes motor neuron disease independent of copper chaperone-mediated copper loading," Nature Neuroscience, vol. 5, no. 4, pp. 301-307, 2002.

[75] A. Pramatarova, J. Laganière, J. Roussel, K. Brisebois, and G. A. Rouleau, "Neuron-specific expression of mutant superoxide dismutase 1 in transgenic mice does not lead to motor impairment," Journal of Neuroscience, vol. 21, no. 10, pp. 3369-3374, 2001.

[76] Y. H. Gong, A. S. Parsadanian, A. Andreeva, W. D. Snider, and J. L. Elliott, "Restricted expression of $\mathrm{G} 86 \mathrm{R} \mathrm{Cu} / \mathrm{Zn}$ superoxide dismutase in astrocytes results in astrocytosis but does not cause motoneuron degeneration," Journal of Neuroscience, vol. 20, no. 2, pp. 660-665, 2000.

[77] L. Wang, K. Sharma, H. X. Deng et al., "Restricted expression of mutant SOD1 in spinal motor neurons and interneurons induces motor neuron pathology," Neurobiology of Disease, vol. 29, no. 3, pp. 400-408, 2008.
[78] S. Boillée, K. Yamanaka, C. S. Lobsiger et al., "Onset and progression in inherited ALS determined by motor neurons and microglia," Science, vol. 312, no. 5778, pp. 1389-1392, 2006.

[79] K. Yamanaka, S. J. Chun, S. Boillee et al., "Astrocytes as determinants of disease progression in inherited amyotrophic lateral sclerosis," Nature Neuroscience, vol. 11, no. 3, pp. 251253, 2008.

[80] L. Wang, D. H. Gutmann, and R. P. Roos, "Astrocyte loss of mutant SOD1 delays ALS disease onset and progression in G85R transgenic mice," Human Molecular Genetics, vol. 19, no. $24,2010$.

[81] C. S. Lobsiger, S. Boillee, M. McAlonis-Downes et al., "Schwann cells expressing dismutase active mutant SOD1 unexpectedly slow disease progression in ALS mice," Proceedings of the National Academy of Sciences of the United States of America, vol. 106, no. 11, pp. 4465-4470, 2009.

[82] T. M. Miller, S. H. Kim, K. Yamanaka et al., "Gene transfer demonstrates that muscle is not a primary target for noncell-autonomous toxicity in familial amyotrophic lateral sclerosis," Proceedings of the National Academy of Sciences of the United States of America, vol. 103, no. 51, pp. 1954619551, 2006.

[83] M. Wong and L. J. Martin, "Skeletal muscle-restricted expression of human SOD1 causes motor neuron degeneration in transgenic mice," Human Molecular Genetics, vol. 19, no. 11, pp. 2284-2302, 2010.

[84] G. Dobrowolny, M. Aucello, E. Rizzuto et al., "Skeletal muscle is a primary target of SOD1-mediated toxicity," Cell Metabolism, vol. 8, no. 5, pp. 425-436, 2008.

[85] S. Zhu, I. G. Stavrovskaya, M. Drozda et al., "Minocycline inhibits cytochrome c release and delays progression of amyotrophic lateral sclerosis in mice," Nature, vol. 417, no. 6884, pp. 74-78, 2002.

[86] L. Van Den Bosch, P. Tilkin, G. Lemmens, and W. Robberecht, "Minocycline delays disease onset and mortality in a transgenic model of ALS," NeuroReport, vol. 13, no. 8, pp. 1067-1070, 2002.

[87] J. Kriz, M. D. Nguyen, and J. P. Julien, "Minocycline slows disease progression in a mouse model of amyotrophic lateral sclerosis," Neurobiology of Disease, vol. 10, no. 3, pp. 268-278, 2002.

[88] P. H. Gordon, D. H. Moore, R. G. Miller et al., "Efficacy of minocycline in patients with amyotrophic lateral sclerosis: a phase III randomised trial," Lancet Neurology, vol. 6, no. 12, pp. 1045-1053, 2007.

[89] M. Benatar, "Lost in translation: treatment trials in the SOD1 mouse and in human ALS," Neurobiology of Disease, vol. 26, no. 1, pp. 1-13, 2007.

[90] S. Scott, J. E. Kranz, J. Cole et al., "Design, power, and interpretation of studies in the standard murine model of ALS," Amyotrophic Lateral Sclerosis, vol. 9, no. 1, pp. 4-15, 2008.

[91] A. C. Ludolph, C. Bendotti, E. Blaugrund et al., "Guidelines for preclinical animal research in ALS/MND: a consensus meeting," Amyotrophic Lateral Sclerosis, vol. 11, no. 1-2, pp. 38-45, 2010.

[92] J. Lladó, C. Haenggeli, A. Pardo et al., "Degeneration of respiratory motor neurons in the SOD1 G93A transgenic rat model of ALS," Neurobiology of Disease, vol. 21, no. 1, pp. 110-118, 2006.

[93] A. C. Lepore, C. Tolmie, J. O’Donnell et al., "Peripheral hyperstimulation alters site of disease onset and course in 
SOD1 rats," Neurobiology of Disease, vol. 39, no. 3, pp. 252264, 2010.

[94] D. M. Ruddy, M. J. Parton, A. Al-Chalabi et al., "Two families with familial amyotrophic lateral sclerosis are linked to a novel locus on chromosome 16q," American Journal of Human Genetics, vol. 73, no. 2, pp. 390-396, 2003.

[95] R. Fujii, S. Okabe, T. Urushido et al., "The RNA binding protein TLS is translocated to dendritic spines by mGluR5 activation and regulates spine morphology," Current Biology, vol. 15, no. 6, pp. 587-593, 2005.

[96] M. Neumann, D. M. Sampathu, L. K. Kwong et al., "Ubiquitinated TDP-43 in frontotemporal lobar degeneration and amyotrophic lateral sclerosis," Science, vol. 314, no. 5796, pp. 130-133, 2006.

[97] C. Lagier-Tourenne and D. W. Cleveland, "Rethinking ALS: the FUS about TDP-43," Cell, vol. 136, no. 6, pp. 1001-1004, 2009.

[98] F. Geser, V. M. Y. Lee, and J. Q. Trojanowski, “Amyotrophic lateral sclerosis and frontotemporal lobar degeneration: a spectrum of TDP-43 proteinopathies," Neuropathology, vol. 30, no. 2, pp. 103-112, 2010.

[99] E. Buratti and F. E. Baralle, "The molecular links between TDP-43 dysfunction and neurodegeneration," Advances in Genetics, vol. 66, pp. 1-34, 2009.

[100] J. R. Levy, C. J. Sumner, J. P. Caviston et al., "A motor neuron disease-associated mutation in p150 perturbs dynactin function and induces protein aggregation," Journal of Cell Biology, vol. 172, no. 5, pp. 733-745, 2006.

[101] A. Hirano, H. Donnenfeld, S. Sasaki, and I. Nakano, "Fine structural observations of neurofilamentous changes in amyotrophic lateral sclerosis," Journal of Neuropathology and Experimental Neurology, vol. 43, no. 5, pp. 461-470, 1984.

[102] G. A. Rouleau, A. W. Clark, K. Rooke et al., "SOD1 mutation is associated with accumulation of neurofilaments in amyotrophic lateral sclerosis," Annals of Neurology, vol. 39, no. 1, pp. 128-131, 1996.

[103] A. Al-Chalabi, P. M. Andersen, P. Nilsson et al., "Deletions of the heavy neurofilament subunit tail in amyotrophic lateral sclerosis," Human Molecular Genetics, vol. 8, no. 2, pp. 157164, 1999.

[104] J. P. Julien, "Amyotrophic lateral sclerosis: unfolding the toxicity of the misfolded," Cell, vol. 104, no. 4, pp. 581-591, 2001.

[105] I. V. Mersiyanova, A. V. Perepelov, A. V. Polyakov et al., "A new variant of Charcot-Marie-Tooth disease type 2 is probably the result of a mutation in the neurofilament-light gene," American Journal of Human Genetics, vol. 67, no. 1, pp. 37-46, 2000.

[106] B. G. Szaro and M. J. Strong, "Post-transcriptional control of neurofilaments: new roles in development, regeneration and neurodegenerative disease," Trends in Neurosciences, vol. 33, no. 1, pp. 27-37, 2010.

[107] Z. Nie, J. Wu, J. Zhai et al., "Untranslated element in neurofilament mRNA has neuropathic effect on motor neurons of transgenic mice," Journal of Neuroscience, vol. 22, no. 17, pp. 7662-7670, 2002.

[108] C. L. Leung, C. Z. He, P. Kaufmann et al., "A pathogenic peripherin gene mutation in a patient with amyotrophic lateral sclerosis," Brain Pathology, vol. 14, no. 3, pp. 290-296, 2004.

[109] F. Gros-Louis, R. Larivière, G. Gowing et al., "A frameshift deletion in peripherin gene associated with amyotrophic lateral sclerosis," Journal of Biological Chemistry, vol. 279, no. 44, pp. 45951-45956, 2004.
[110] Y. Masu, E. Wolf, B. Holtmann, M. Sendtner, G. Brem, and $\mathrm{H}$. Thoenen, "Disruption of the CNTF gene results in motor neuron degeneration," Nature, vol. 365, no. 6441, pp. 27-32, 1993.

[111] V. M. Y. Lee, M. Goedert, and J. Q. Trojanowski, "Neurodegenerative tauopathies," Annual Review of Neuroscience, vol. 24, pp. 1121-1159, 2001.

[112] I. Taes, A. Goris, R. Lemmens et al., "Tau levels do not influence human ALS or motor neuron degeneration in the SOD1G93A mouse," Neurology, vol. 74, no. 21, pp. 16871693, 2010. 


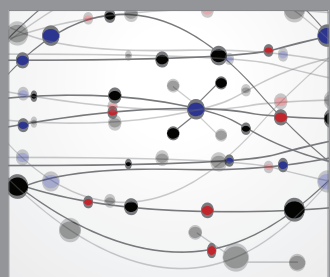

The Scientific World Journal
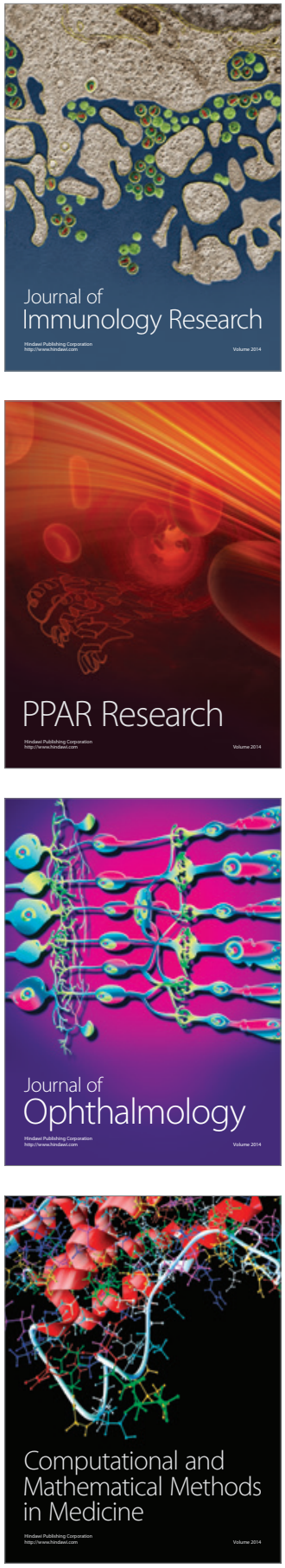

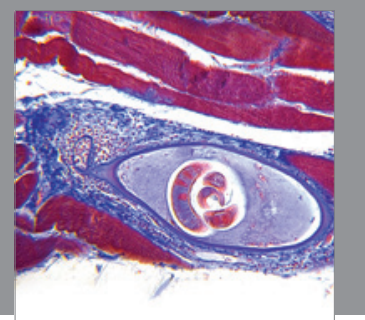

Gastroenterology

Research and Practice
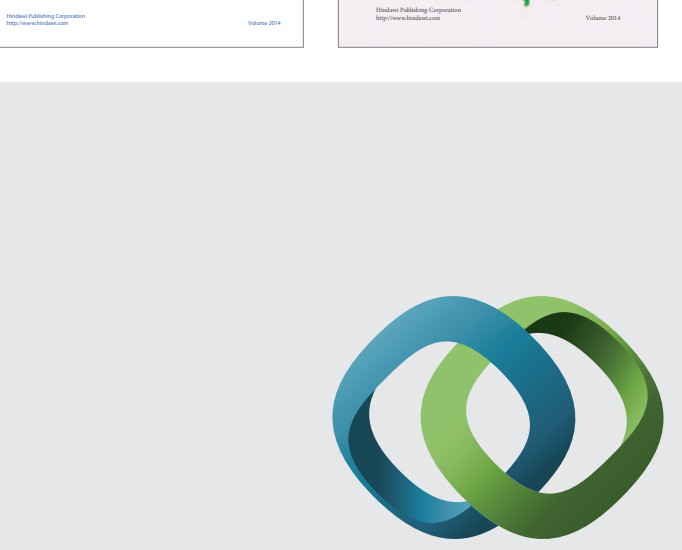

\section{Hindawi}

Submit your manuscripts at

http://www.hindawi.com
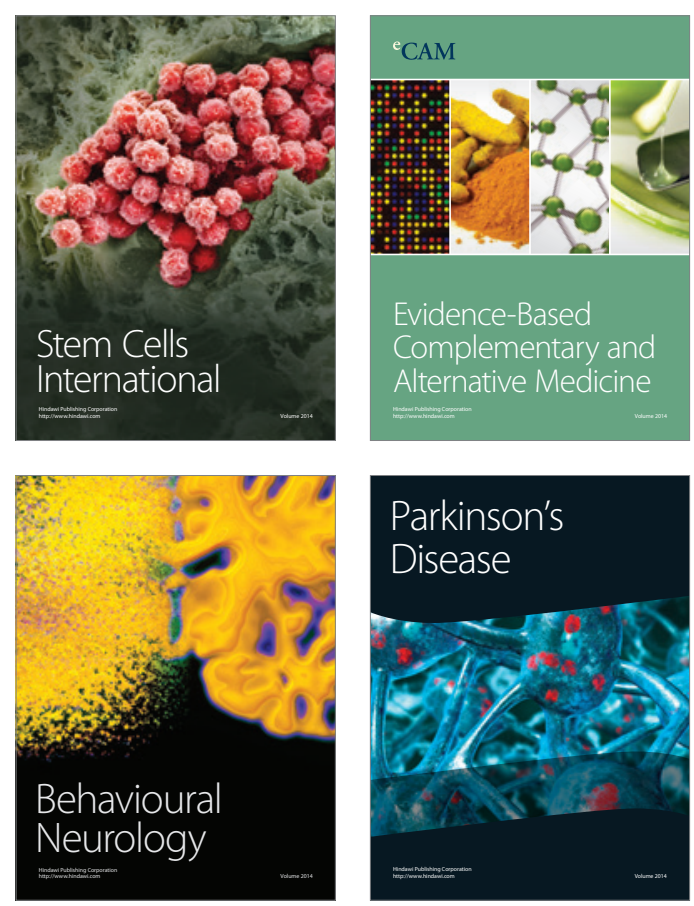

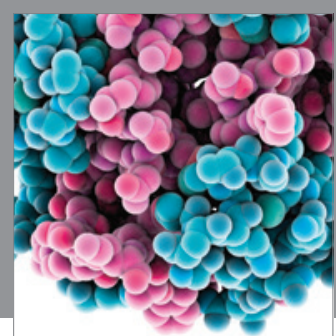

Journal of
Diabetes Research

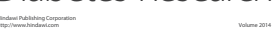

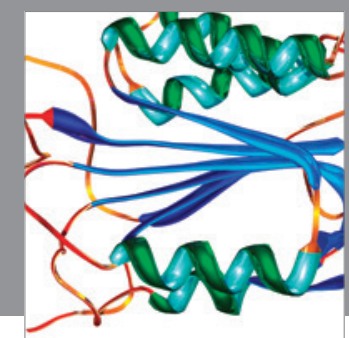

Disease Markers
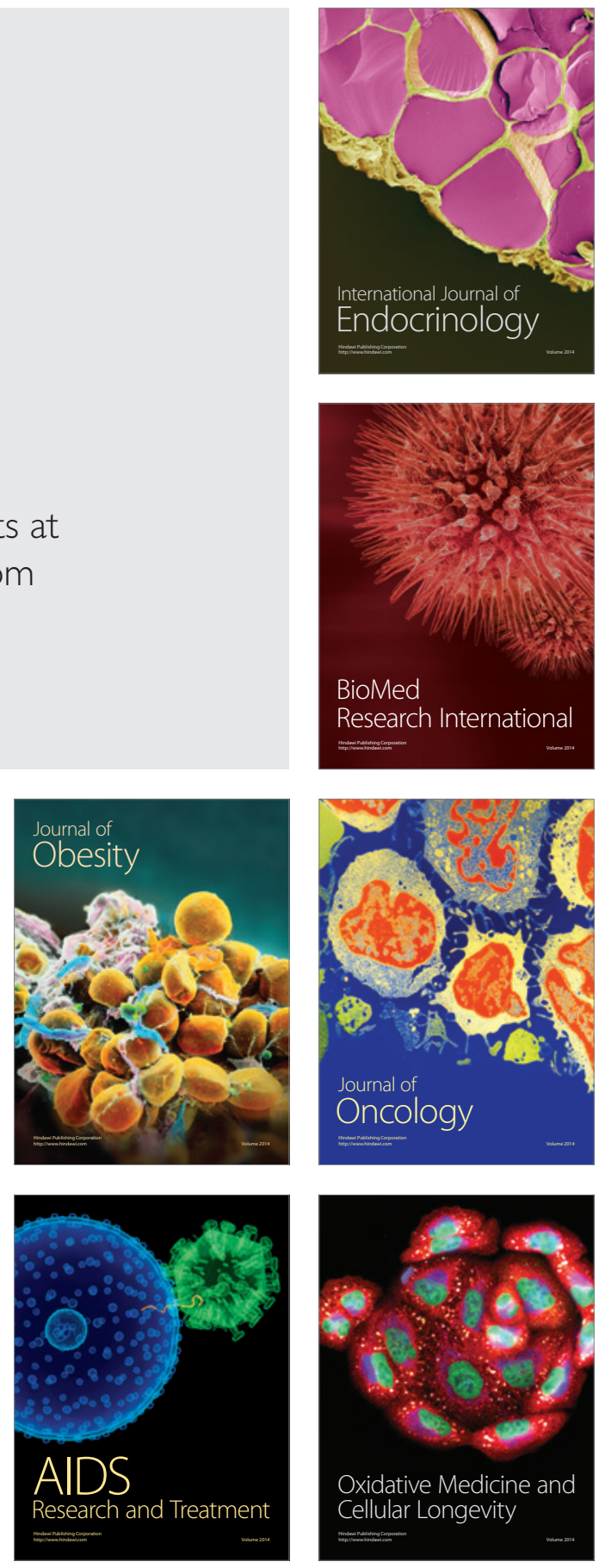